\title{
Effects of Active Oxygen Scavengers on the Peroxidation of Linoleic Acid Catalyzed by Dehydro-L-Ascorbic Acid or Its Degradation Products
}

\author{
Masanosuke TAKAgI, Hiroshi OnODERA, Ikuo MiYAmoto, \\ and Naofumi MORITA ${ }^{1}$ \\ Department of Agricultural Chemistry, College of Agriculture, \\ University of Osaka Prefecture, \\ Sakai, Osaka 591, Japan \\ (Received December 5, 1987)
}

\begin{abstract}
Summary The addition of 1,4-diazabicyclo-[2,2,2]octane (DABCO) (100 mM) or 1,2,-dihydroxybenzene-3,5-disulfonic acid (Tiron) (1 mM) to a reaction mixture containing $10 \mathrm{~mm}$ linoleic acid (LA), $20 \% \mathrm{EtOH}$, and $135 \mu \mathrm{M}$ dehydroascorbic acid (DHA) as a catalyst suppressed LA peroxidation, but the addition of mannitol $(\sim 100 \mathrm{~mm})$, uric acid $(100 \mu \mathrm{M})$, and catalase (6.5 units) did not. DHA or 2,3-diketo-L-gulonic acid (DKG) accelerated LA peroxidation, but the splitting products of DHA did not affect LA peroxidation. These results suggest that some specific radicals are liberated in the degradation of DHA or DKG.
\end{abstract}

Key Words lipid peroxidation, dehydroascorbic acid, active oxygen scavengers, DABCO, Tiron, 2,3-diketogulonic acid

L-Ascorbic acid (AsA) is often added to various foods, beverages, and soft drinks as an antioxidant and antibrowning agent $(1,2)$. However, it is also true that AsA accelerates the peroxidation or deterioration of lipids (3-5). As we reported previously.(5), AsA in the reaction mixture containing 10\% EtOH accelerated linoleic acid (LA) peroxidation, but AsA in $20 \%$ EtOH suppressed the LA peroxidation. This oxidation of LA may be caused by radical species derived from dehydro-L-ascorbic acid (DHA) which is an oxidized form of AsA, or a further degraded product, i.e., 2,3-diketo-L-gulonic acid (DKG)(6). Though the lactone ring of DHA is simply considered to be split to DKG in a weakly alkaline solution, as reported by Kagawa et al. (7), these processes of degradation are still ambiguous. And also, no systematic work has been done on the degraded products of AsA, such as DHA, DKG $(8)$, threonic acid, and oxalic acid $(7,9)$. Since DHA is considered to accelerate LA peroxidation, we are aiming to understand the behavior of active oxygen scavenging agents against DHA.

\footnotetext{
1 高木正之助，小野寺裕，宮本郁生，森田尚文
} 
In this paper, some active oxygen scavenging agents were tested for LA peroxidation catalyzed by oxidation products of AsA. We also tried to understand the involvement of active oxygen radical species in LA peroxidation.

\section{MATERIALS AND METHODS}

Chemicals. LA was purchased from Sigma Chemical Co., diluted to $100 \mathrm{~mm}$ with distilled EtOH under $\mathrm{N}_{2}$ bubbling, and stored at $-20^{\circ} \mathrm{C}$ until use. AsA was obtained from Wako Pure Chemical Industries. DHA was prepared from AsA as reported previously $(5,10)$. DKG was prepared as a $\mathrm{K}$-salt as reported by Kagawa et al. (7). Triose-reductone was kindly donated by Dr. S. Taniguchi of the Radiation Center of Osaka Prefecture. Catalase and 1,4-diazabicyclo-[2,2,2]octane (DABCO) were obtained from Wako Pure Chemical Industries. The 1,2-dihydroxybenzene3,5-disulfonic acid was obtained from Dojin Chemical Laboratories. Other reagents were of analytical grade. Furoic acid (FA) and 3-hydroxy-2-pyrone (HP) were prepared from DHA (11).

Analytical method. The method for the analysis of LA peroxidation was the same as reported before (5). For the case of $10 \mathrm{~mm} \mathrm{LA} \mathrm{containining} 20 \% \mathrm{EtOH}$, $0.5 \mathrm{ml}$ of $100 \mathrm{~mm} \mathrm{LA}, 0.5 \mathrm{ml}$ of EtOH, $4 \mathrm{ml}$ of $0.1 \mathrm{M}$ phosphate buffer (pH 6.8), and $0.1 \mathrm{ml}$ of aqueous solution of AsA or its oxidized product were incubated at $37^{\circ} \mathrm{C}$ with shaking.

A portion $(0.3 \mathrm{ml})$ of the reaction mixture was withdrawn at specified intervals, and $3 \mathrm{ml}$ of $60 \%$ EtOH aqueous solution containing $200 \mathrm{ppm}$ of EDTA was added to the solution. The UV absorption spectrum was measured over the range of 225 $300 \mathrm{~nm}$ with a Hitachi Model 200-10 spectrophotometer. The responses at $233 \mathrm{~nm}$ and $265 \mathrm{~nm}$ were taken for the measurement of LA hydroperoxide formed and AsA remaining, respectively.

\section{RESULTS AND DISCUSSION}

\section{Effects of DABCO on $L A$ peroxidation}

Figure 1 shows the results of a singlet oxygen scavenging agent on the peroxidation of LA catalyzed by AsA or DHA. With lower concentrations (5 or $10 \mathrm{~mm}$ ) of DABCO, no effective suppression of LA peroxidation was observed, but with higher concentrations (50 or $100 \mathrm{~mm}$ ) of DABCO, the suppression of LA peroxidation was observed at the initial stage of the reaction. Thereafter, it gradually turned to an acceleration of LA peroxidation. However, the degree of suppression was not necessarily enough, as judged from the concentration of DABCO in the reaction mixture. Therefore, this suppression of LA peroxidation in the initial stage of the reaction may not be caused by scavenging of singlet oxygen as reported (12), but may be due to other causes. 


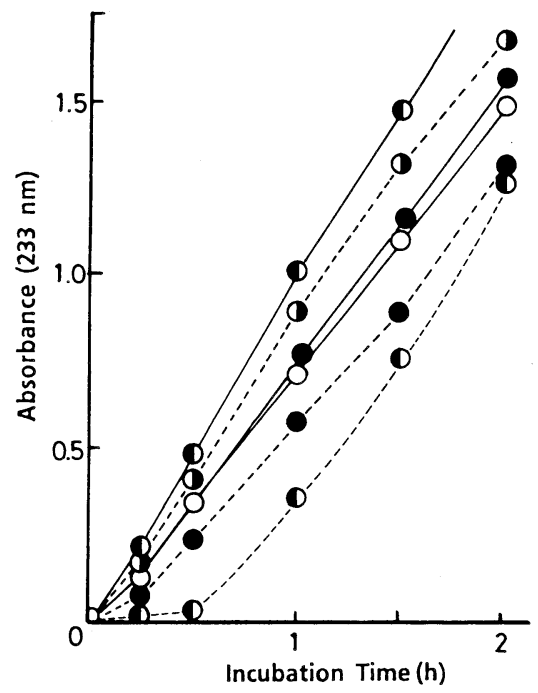

Fig. 1 .

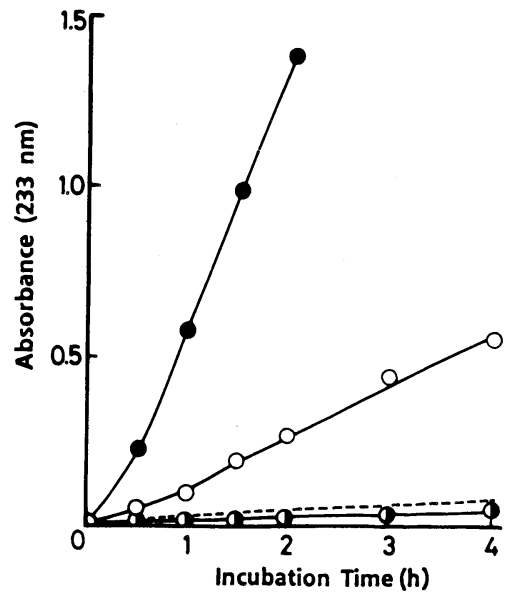

Fig. 2.

Fig. 1. Effects of DABCO concentrations on LA peroxidation catalyzed by DHA. The reaction mixture contained $10 \mathrm{~mm} \mathrm{LA}, 135 \mu \mathrm{M} \mathrm{DHA}, 20 \% \mathrm{EtOH}$, and various concentrations of DABCO in $80 \mathrm{~mm}$ phosphate buffer $(\mathrm{pH} 7)$. It was incubated at $37^{\circ} \mathrm{C}$ with shaking. A sample $(0.3 \mathrm{ml})$ of the reaction mixture was withdrawn and added to $3 \mathrm{ml}$ of $60 \%$ EtOH solution containing EDTA (200 ppm), and the UV absorption spectra of the solution was measured. Concn. of DABCO: $-\mathrm{O}-, 0 \mathrm{~mm}$

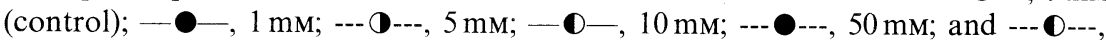
$100 \mathrm{~mm}$.

Fig. 2. Effects of Tiron concentrations on LA peroxidation catalyzed by DHA. The reaction mixture contained $135 \mu \mathrm{M}$ DHA, $10 \mathrm{~mm} \mathrm{LA,} 20 \% \mathrm{EtOH}$, and various concentrations of Tiron in $80 \mathrm{~mm}$ phosphate buffer ( $\mathrm{pH}$ 7). The broken line shows the control values without a catalyst. Other conditions were the same as for Fig.

1. Concn. of Tiron: ๑, $0 \mathrm{~mm}$;, $0.1 \mathrm{~mm}$; $1 \mathrm{~mm}$.

\section{Effects of Tiron on LA peroxidation}

A superoxide scavenging agent, Tiron, was tested on LA peroxidation catalyzed by DHA (Fig. 2). Additions of $0.1 \mathrm{~mm}$ or $1 \mathrm{~mm}$ Tiron suppressed LA peroxidation; Tiron scavenged the superoxide radical generated from a step in the degradation pathway of DHA. Therefore, no positive acceleration of LA peroxidation was observed. Especially, in the case of $1 \mathrm{~mm}$ Tiron, the degree of suppression of LA peroxidation was complete and was greater than that of the control value without DHA, which is shown in Fig. 2 as a broken line.

\section{Effects of mannitol on LA peroxidation catalyzed by DHA}

Figure 3 shows the effects on mannitol, an $\cdot \mathrm{OH}$ scavenging agent, on LA peroxidation catalyzed by DHA. The addition of mannitol ( $\sim 100 \mathrm{~mm})$ only slightly 


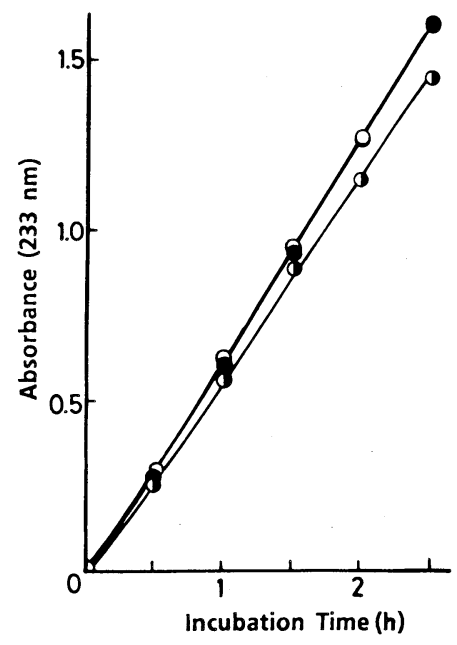

Fig. 3.

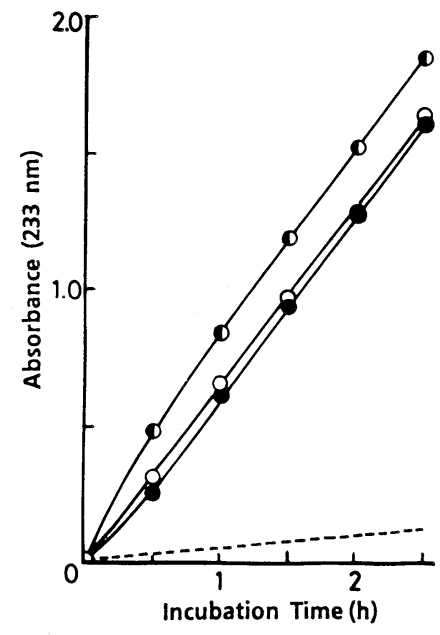

Fig. 4.

Fig. 3. Effects of mannitol concentration on LA peroxidation. The reaction mixture was the same as for Fig. 1, except that mannitol was used instead for DABCO. Concn. of mannitol: $0,0 \mathrm{~mm}$; O, $10 \mathrm{~mm}$; and $100 \mathrm{~mm}$.

Fig. 4. Effects of catalase concentrations on LA peroxidation catalyzed by DHA. The reaction mixture was the same as for Fig. 1 except for the catalase. Concn. of catalase: $\bigcirc, 0 \mu \mathrm{g} / \mathrm{ml} ; \bigcirc, 1 \mu \mathrm{g} / \mathrm{ml}(0.65 \mathrm{U})$; and $\boldsymbol{O}, 10 \mu \mathrm{g} / \mathrm{ml}(6.5 \mathrm{U})$. The broken line shows the control values without a catalyst.

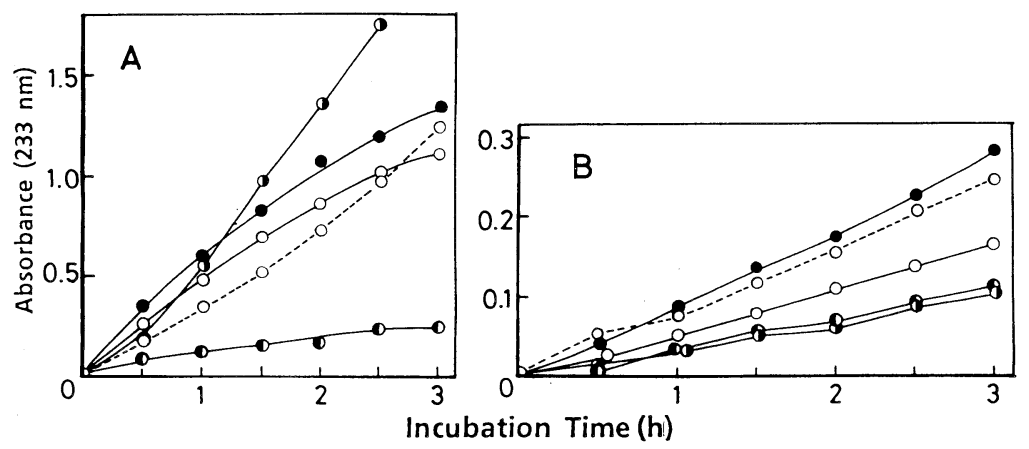

Fig. 5. Effects of alcohols on LA peroxidation catalyzed by DHA. The reaction mixture contained $135 \mu \mathrm{M}$ DHA, $10 \mathrm{~mm}$ LA, and various kinds of $20 \%$ alcohol in $80 \mathrm{~mm}$ phosphate buffer ( $\mathrm{pH} 7)(\mathrm{A})$ or acetate buffer $(\mathrm{pH} 4.0)(\mathrm{B})$. Other conditions

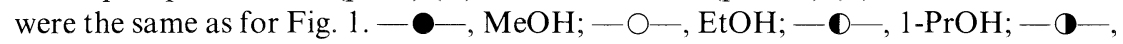
2-PrOH; --- $\mathrm{O}---, t-\mathrm{BuOH}$.

suppressed LA peroxidation, but not significantly. Under the reaction conditions used, the potency of EtOH as an $\mathrm{OH}$ radical scavenging agent might be greater than that of mannitol. Therefore, the behavior of mannitol as a scavenger of $\mathrm{OH}$ radicals 
cannot be distingished from the similar effect of $\mathrm{EtOH}$ contained in the reaction mixture.

\section{Effects of catalase on LA peroxidation catalyzed by $D H A$}

Figure 4 shows the effects of catalase on LA peroxidation catalyzed by DHA. The addition of catalase (6.5 units) accelerated the LA peroxidation faster than that of the control without catalase. Here, catalase did not suppress the peroxidation of LA, but accelerated the reaction. This may be due to a basic property of catalase as an enzyme having a heme group. In an in vivo experiment, $\mathrm{H}_{2} \mathrm{O}_{2}$, one of the active forms of oxygen, may be scavenged by ascorbate peroxidase, not by catalase, as reported by Dalton et al. (13).

\section{Effects of uric acid on LA peroxidation catalyzed by DHA}

Uric acid is known to suppress the peroxidation of lipids in vivo. The effects of uric acid on LA peroxidation catalyzed by $135 \mu \mathrm{M}$ DHA were tested in a reaction mixture (pH 7) containing 20\% EtOH and $100 \mu \mathrm{M}$ uric acid. In the results, we could not observe distinct suppression of LA peroxidation upon the addition of $135 \mu \mathrm{M}$ DHA. Since uric acid is only slightly soluble in water, we could not test its higher concentrations.

\section{Effects of alcohols on LA peroxidation catalyzed by $D H A$}

Since tert-butyl alcohol $(t-\mathrm{BuOH})$ and 2-propyl alcohol $(2-\mathrm{PrOH})$ are scavengers for $\cdot \mathrm{OH}$ like $\mathrm{EtOH}$, we tested the effects of alcohol on LA peroxidation using these alcohols, including primary alcohols (Fig. 5). In acidic solutions (pH 4), both 1-PrOH and 2-PrOH had almost the same behavior on LA peroxidation, but in neutral reaction mixtures they caused a rapid peroxidation of $\mathrm{LA}$, and the degree of acceleration for LA peroxidation was faster in $n-\mathrm{PrOH}$ than in $t-\mathrm{BuOH}$. As is evident from Fig. 5, the higher the polarity of the alcohol used, the greater the acceleration of LA peroxidation(5), and secondary or tertiary alcohols had more potency in the acceleration of LA peroxidation. Furthermore, no distinct acceleration on LA peroxidation was observed in acidic $\mathrm{pH}$ solution, but a significant degree of LA peroxidation was observed in neutral $\mathrm{pH}$ solutions.

\section{Effects of $p H$ on $L A$ peroxidation}

Since the rate of degradation or autoxidation of AsA or DHA might be lower in acidic $\mathrm{pH}$ solutions than in neutral solutions, we tested LA peroxidation catalyzed by DHA in acetate buffer ( $\mathrm{pH}$ 4) (data not shown). The rate of LA peroxidation was quite slow, as expected. As LA could not disperse homogeneously in this acidic solution containing $20 \% \mathrm{EtOH}$, this aggregation might retard or suppress the acceleration of LA peroxidation. This may suggest that active-oxygen radicals are less formed in this acidic buffer solution than in neutral media, since DHA is rather stable in acidic solutions. 


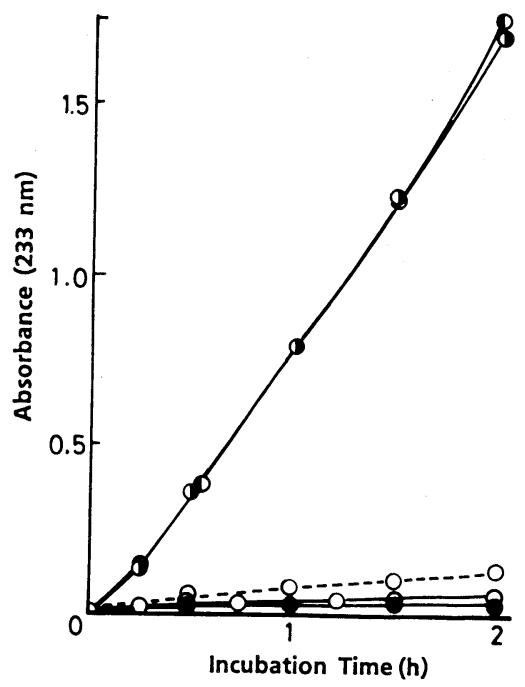

Fig. 6. Effects of degraded products of DHA on LA peroxidation. The reaction mixture was the same as for Fig. 1 except for the catalyst. - $\mathrm{O}-$, no catalyst; -—, $135 \mu \mathrm{M}$ DHA; —- $135 \mu \mathrm{M}$ DKG; - - $135 \mu \mathrm{M}$ 3-hydroxy-2-pyrone; --- $\bigcirc--, 135 \mu \mathrm{M}$ threonic acid.

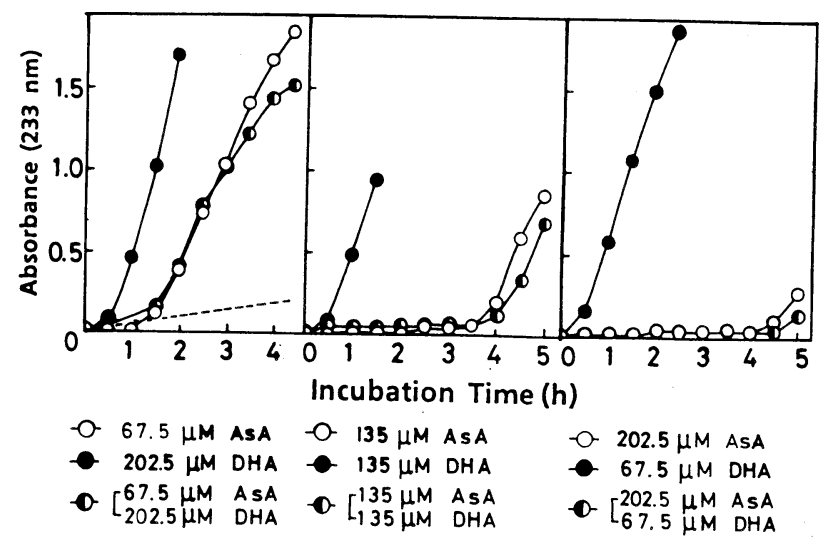

Fig. 7. Combination of different concentrations of AsA and DHA on LA peroxidation. The reaction conditions were the same as for Fig. 1 except the combination of the catalyst. The broken line shows the control values without catalysts.

Other scavengers for $L A$ peroxidation

The effects of 3(2)-tert-butyl-4-hydroxyanisole (BHA) on LA peroxidation catalyzed by DHA were tested (data not shown). Addition of BHA $(10 \mu \mathrm{M})$ to the reaction mixture containing $135 \mu \mathrm{M}$ DHA and $10 \mathrm{~mm} \mathrm{LA}$ in $20 \% \mathrm{EtOH}$ suppressed 
the LA peroxidation very clearly: the degree of suppression was complete, compared with that of DHA as the control.

\section{Effects of degraded products of DHA on LA peroxidation (8)}

Threonic acid, oxalic acid, HP, and FA were tested for their effects on LA peroxidation with $20 \% \mathrm{EtOH}$ in the reaction mixture (Fig. 6). None of these materials caused any positive acceleration of LA peroxidation. This suggests that once the carbon-carbon linkage of DHA is split, LA peroxidation could not be accelerated. When DKG $(135 \mu \mathrm{M})$ was added to the reaction mixture instead of DHA, no distinct differences were observed between the agents.

\section{Effects of combinations of AsA and DHA on LA peroxidation}

The combined effects of AsA and DHA on LA peroxidation were tested by changing the ratio from one- to three-fold (Fig. 7). The behavior of suppression of LA peroxidation caused by AsA was quite different from that caused by DHA. That is, additions of $67.5 \mu \mathrm{m} \mathrm{AsA}$ and $202.5 \mu \mathrm{m}$ DHA had effects on LA peroxidation similar to that caused by $67.5 \mu \mathrm{M}$ AsA only. But after incubation for $3 \mathrm{~h}$, the degree of suppression of LA peroxidation became more distinct than that caused by AsA only. Additions of equal amounts (135 $\mu \mathrm{m}$ each) of AsA and DHA to the reaction mixture retarded the LA peroxidation distinctly. There was no significant difference in the response of LA peroxidation between AsA only and AsA plus DHA in the early stage of incubation. But, after $4 \mathrm{~h}$ of incubation, the LA peroxidation caused by AsA and DHA was suppressed more clearly than that caused by AsA. With $202.5 \mu \mathrm{M} \mathrm{AsA}$ and $67.5 \mu \mathrm{M}$ DHA, the suppressive effect of LA peroxidation was similar to that of $202.5 \mu \mathrm{M}$ AsA except that the duration of the suppression of LA peroxidation was a little longer. These results suggest that during the presence of AsA as a proton donor in the reaction mixture, the scavenging action derived from some further degradation products of DHA or DKG might be involved.

In the case of combinations of AsA and DHA, the LA peroxidation was suppressed regardless of the ratio of AsA and DHA which was added to the reaction mixture. Even if the content of AsA was less than that of DHA, the degree of suppression of LA peroxidation catalyzed by AsA including DHA was more potent than that of AsA only. This is an interesting phenomenon with regard to understanding the mechanism of radical formation or the scavenging action of radical species.

In these results, BHA and Tiron were found to be the most potent agents for the scavenging of radical species derived from DHA. Since BHA has an antioxidative potency in hydrogenated vegetable oil or animal oil because of the hydrophobic properties of the agent, as reported $(14,15)$, the suppression of LA peroxidation catalyzed by DHA may be attributed to the same mechanism. As a consequence. DHA and DKG, which are oxidized forms of AsA, or further degraded products definitely catalyze the LA peroxidation. However, since no distinct acceleration of the LA peroxidation was observed when the carbon-carbon 
linkage of DKG or DHA was split, some kinds of active oxygen species, such as $\mathrm{R}^{*}$ or $\mathrm{O}_{2}{ }^{-}$, may be formed by the transformation and subsequent enolization of DKG, as reported by Otsuka et al. (6). From the polarographic studies, the presence of a more oxidizable material than AsA could be pertinent to these problems (16).

From our results, it is clear that some kinds of radical species are formed from the degradation of the 3,4-endiol form of DKG. However, the relationships between the degradation of these compounds and the formation and reaction of $\mathrm{O}_{2}{ }^{-}$ or $\mathrm{R}^{*}$ radicals require further investigation.

\section{REFERENCES}

1) Smoot, J. M., and Nagy, S. (1980): Effect of storage temperature and duration on total vitamin C content of canned single-strength grapefruit juice. J. Agric. Food Chem., 28, 417-421.

2) Horowitz. I., Fabry, E. M., and Gerson, C. O. (1976): Bioavailability of ascorbic acid in orange juice. J. Am Med. Assoc., 235, 2624-2625.

3) Kanner, J., and Mendel, H. (1977): Proxidant and antioxidant effects of ascorbic acid and metal salts in a beta-carotene-linoleate model system. J. Food Sci., 42, 60-64.

4) Kunimoto, M., Inoue, K., and Nojima, S. (1981): Effect of ferrous ion and ascorbateinduced lipid peroxidation on liposomal membranes. Biochim. Biophys. Acta, 644. 169178.

5) Takagi, M., Higashioka, H., Tamura, K., and Morita. N. (1986): Effect of L-ascorbic acid and dehydro-L-ascorbic acid on the peroxidation of linoleic acid in neutral phosphate buffer cotaining alcohol. Agric. Biol. Chem., 50, 41-47.

6) Otsuka, M., Kurata, T., and Arakawa, N. (1986): Isolation and characterization of a degradation product derived from 2,3-diketo-L-gulonic acid, in Amino-Carbonyl Reactions in Food and Biological Systems, eds. by Fujimaki, M., Namiki, M., and Kato, H., Developments in Food Science, Vol. 13, Elsevier, Amsterdam, pp. 77-84

7) Kagawa, Y. (1962): Enzymatic studies on L-ascorbic acid catabolism in animal. $J$. Biochem., 51, 134-144.

8) Kurata, T. (1976): Mechanism of degradation and discoloration reaction of L-ascorbic acid. Nippon Nōgeikagaku Kaishi (in Japanese), 50, R209-216.

9) Sawyer, D. T., Chiericato, G. C., Jr., and Tsuchiya, T. (1982): Oxidation of ascorbic acid and dehydroascorbic acid by superoxide ion in aprotic media. J. Am. Chem. Soc., 104, 6273-6278.

10) Takagi, M., Higashioka, H., and Morita, N. (1986): Effects of lipophilic derivatives of L-ascorbic acid and dehydro-L-ascorbic acid on the peroxidation of linoleic acid in neutral phosphate buffer containing alcohol. J. Nutr. Sci. Vitaminol., 32, 389-394.

11) Takagi, M., Kawajiri, A., Morita, N., Sawada, S., and Mizunami, Y. (1987): Electrochemical properties of degraded products from dehydroascorbic acid in neutral and weakly acidic solutions. Abstracts of papers, The Annual Meeting of Agricultural Chemical Society of Japan, p. 784.

12) Nathan, C. F., Silverstein, S. G., Brukner, L. H., and Cohn, Z. A. (1979): Extracellular cytosis by activated macrophages and granulocytes. J. Exp. Med., 149, 100-113.

13) Dalton, D. A., Hanus, F. J., Russell, S. A., and Evans, H. J. (1987): Purification, properties, and distribution of ascorbate peroxidase in Legume root nodules. Plant Physiol., 83, 789-794. 
14) Pongracz, G. (1973): Antioxidant mixtures for use in food. Int. J. Vitam. Nutr. Res., 43, 517-525.

15) Slaga, T. J., and Bracken, W. M. (1977): The effect of antioxidants on skin tumor inhibition and aryl hydrocarbon hydroxylase. Cancer Res., 37, 1631-1635.

16) Takagi, M., Morita, N., Kawajiri, A., Sasai, K., and Sawada, S. (1987) Electrochemical characteristics of a reductant derived from dehydroascorbic acid or 2,3-diketogulonic acid in neutral buffer solution. Rev. Polarogr., 33, 65. 